\title{
Minka Stoyanova
}

\section{GAMING SYSTEMS: CREATIVE CRITIQUES OF THE LUDIC REAL}

APRJA Volume 4, Issue 1, 2015

ISSN 2245-7755

CC license: 'Attribution-NonCommercial-ShareAlike'. 
A headline on the popular blogging site, medium.com, reads: "Cruel Intentions: How I hacked Tinder and got 2015 matches in under 17 hours (the formula to become wanted on Tinder)." Miranda July has released an app and associated video - funded by fashion brand, MiuMiu - that allows users to select physical proxies to deliver (text-)messages. These playful actions are only two examples of how creative individuals are critiquing the process by which the ubiquitous adoption of mobile computing devices has implicated all of us in a techno-social system of interaction dominated by the codified and computational logic of the game. These 'creatives' reveal inherent dissonances in the computational structures we have accepted by extending the game logic to its (often) absurdist conclusions, thus allowing us to achieve an essential critical distance and inviting us to question the validity of those structures.

The following essay will first examine the modes by which these computational structures, in the guise of games, have come to dominate our understanding of, and interaction with, the non-game world. It will then identify how the application of this logic creates cognitive and phenomenological ruptures, which can be leveraged by creative individuals to reveal logical fallacies within the applied structures. Throughout, it will identify and analyze creative practices that exemplify responses to these logical fallacies in order to identify ways in which a new class of creative individuals is emerging to tackle the dangerous slippage between gamespace (the space of play, games) and game-ic (gamic) space (ordinary/real life to which ludic properties have been applied).

\section{Gamification: from} Mary Poppins to slippery signifiers

Play theorists Johann Huizinga and Roger Caillois both situate play outside of the real or the ordinary. Play, for Huizinga, occurs within "certain fixed limits of time and place", within what he terms, the "magic circle" (Huizinga 28; Caillois 9-10). However, as the logic of the game and the attitude of play (the ludic) permeate our daily lives, this boundary becomes increasingly permeable. The process of 'gamification' - the application of game-like structures to non-game activity - is one mode by which the logic of games encroaches upon our experience of both the playful and the serious, and by which the two are becoming increasingly indistinguishable. The first, and most obvious example of gamification is the application of rewards or competition to labour activities in order to incentivize production. The "whistlewhile-you-work" or "Mary Poppins approach [1] to personal motivation is a well-known and historical model of personal behavior modification, so it is not surprising that it has its analogue in the modern digital age. Gamified quantified-self applications such as fitness and dietary trackers, work tracking applications such as pomodoro timers, Written Kitten or Write or Die exemplify this approach to personal behaviour modification and point to its growing popularity.

However, it is not simply the application of incentive-based logic that is driving the gamic turn in digital technologies. Mobile computational systems which are becoming increasingly coterminous with our physical bodies, through the application of hegemonic interaction design standards are making it increasingly difficult to differentiate the 'magic circle' of play (Huizinga) from the fully 
serious realm of work or 'ordinary life'. For instance, augmented reality systems (such as Google Glass) introduce a mode of interaction that once iconically typified gamespace, the heads-up-display (HUD).[2] In video games the HUD feels "uncomfortable in its 2-dimensionality" (Galloway 35 ). Its inability to visually penetrate the core of the rendered game world - the fact that the HUD creates a screen through which the game world is viewed and which sits permanently on top of, and thus external to, the rendered game world - acts as a constant visual reminder of our presence in a space which is outside of the real or the ordinary; 'a magic circle'. However, if augmented reality technologies like Google Glass continue to increase in popularity, what once signified the fiction of our experience might become a dominant mode of interaction with the real, thus drawing the experience of the real closer to that of the game. Conversely, improved virtual reality technology like the Oculus Rift promises an increasingly real-like experience of gameplay through its fully immersive interface, bringing the experience of the game closer to that of the real.

In Critical Technology, Graeme Kirkpatrick outlines three ways by which games have driven the overall trajectory of technological development (83). The first of these influences has arisen through the introduction, normalization and subsequent proliferation of hegemonic human-computer interaction design standards, visual shorthands such as the aforementioned HUD. Secondly, in order to allow for the implementation of these globally adopted design tropes as well as in order to continuously increase the spectacularity of games, game systems have driven technological advancements such as increased computational power. Finally, games have driven a conception of technology as a source of "friendly, exciting and 'fun' illusions" (83-84). In this final aspect,
Kirkpatrick notes that "games train people for life in a society dominated by computer technology" (84).[3]

However, as the phenomenological experience of game-play and that of ordinarylife become increasingly indistinguishable we create the conditions for a cognitive slippage between the two. In other words, it becomes increasingly difficult for individuals to distinguish between immersive or pervasive gamespace and gamic space - ordinary life that bears a resemblance to gamespace due to its adoption of certain game elements.

\section{From man-playing to the reification of the algorithm}

It is, at this point, of some importance to disambiguate the relationship between 'play' and 'games'. Play is an innate function of humanity, as Huizinga asserts in the forward to his aptly titled, Homo Ludens, man-playing (1). This title is meant to parallel historical and philosophical tropes of human as thinker and/or human as maker. Play, for Huizinga, is of equal importance to both in the philosophical construction of that which humans do, that which defines them, or that which is axiomatic of them as human (1). However, Huizinga is not content to attribute all of the activities of man to the concept of play, to "call all human activity play". For him, such an assertion, while an "ancient wisdom", is nonetheless a "little cheap" (ix). Thus, the thesis of Huizinga's project was not to attribute play to all elements of human life, but instead to explore the play-factor inherent to, and of, culture. As such, "his work is not a study of games, but an inquiry into the creative quality of the play principle in the domain of culture" (Caillois 4). 
A game, on the other hand, is a highly specific form of organized play. While Huizinga regularly uses games as examples of, or as analogies for the play principle, he does not create a clear taxonomy of the rule-bound and highly structured nature of the game. However, it is this rule-bound and structured aspect of the game that allows for the third mode by which gamespace and gamic space converge, as it is the specific structural logic of games that can be ported to non-game experiences, and thus establishes the conditions for the phenomenological ambiguity between the game and the ordinary world made over as game.

Thus, in a bit of foreshadowing, let us consider a description of games which owes more to the field of mathematical game theory than to the philosophy of culture or the social sciences. In his consideration of pervasive games,[4] Bo Kampmann Walther, drawing upon economic game theory, defines games as having "three key mechanisms: absolute rules, contingent strategies, and possible interaction patterns" (249). Any single instance of a game is a manifestation of one interaction pattern as determined by the combination of the game's rules and the player's strategies. This analysis of the structure of the game results in the following 'ontological' definition: "Game play is the actualization of a specific stratification of rules, strategies, and interaction as well as the realization of a certain amalgamation of commands, plans, and paths" (Walther 250). In this definition, rules do not simply prescribe the actions a player might take within a game, they also define the closed environment in which the game occurs (thus, excluding the world outside of the game). Extending this logic to understand that any single instance of a game is one of a given set of permutations made possible by the binding rule-set, Walther is able to assert that "there can be no game world without game rules" (251). In this way, rules structure both the mode of play as well as the game world itself.

\section{Reality made over as 'game'}

'The game of life', 'the rat race', 'the dating game'... games are a well-worn (arguably clichéd) metaphor for our lived experience. Economic game theory (along with its extension into the social sciences) extends this metaphor and reflexively reapplies it in order to determine possible outcomes in various prescribed scenarios. Through the highly mathematical study of player behavior in actual games, game theory attempts to devise a means by which individuals' actions in real life might be predicted based on those individuals' characterization as rational actors within an algorithmically describable scenario space. Without going into the mathematical specifics of economic game theory, as they are largely unimportant within this context, this generally accepted conception of the world as algorithmically describable, and of individuals as rational actors, reflects what Katherine Hayles terms the platonic backhand; the philosophical/theoretical move from noisy multiplicity to abstracted simplicity (12). Hayles recognizes that such "abstraction is of course an essential component in all theorizing, for no theory can account for the infinite multiplicity of our interaction with the real" (12). She warns, however, of the "variegated leaves" and "fractal branchings" that we lose sight of in the process of such abstraction. For Hayles, the danger in such abstraction is introduced when we view the abstraction as the axiomatic model of the more complex reality. When this occurs "complexity appears as a 'fuzzing-up' of an essential reality rather than as a manifestation of the world's holistic nature" (12). 
However, this process of assuming the primacy of the simplified model is the process by which game-like systems (such as Tinder) create virtual manifestations of real world scenarios. Furthermore, in a distinctly modern extension of the application of this logic, these systems create the conditions for what Hayles dubs, the platonic forehand (12): "This move starts from simplified abstraction and, using simulation techniques [...] evolves a multiplicity sufficiently complex that it can be seen as a world of its own" (12). It is towards this process that Kevin Slavin, particularly in reference to financial and economic systems, refers when he describes "how algorithms shape our world". Referring back to Walther's description of the primacy of rules in the building of gamespace, it should be apparent then that the mode by which rules shape game space is at least analogous to the mode by which simplified models of real space, extracted via the study of game systems, have come to shape our real experience.

The dangers of the platonic backhand, the inability of simplified systems in economics, social science or politics to capture or account for all possible cases of reality is well known (Hayles; Gray; Caillois; Taleb). While Nassim Nicholas Taleb refers to the inability of the mathematics of games to appropriately account for the chance of real-life as the "ludic fallacy" (125), Chris Hables Gray notes that "our models of reality are always in tension with reality itself; a model cannot match reality, which is too complex to predict consistently" (29).

And thus, we arrive at the first innate cognitive dissonance that emerges as a result of the slippage between gamespace and gamic space. It is the dissonance resulting from our (often) misguided believe that these simulations are able to accurately reflect the complexity of reality. McKenzie Wark's Gamer Theory is built out of his observation that reality, the world outside of the game, must appear to the gamer as an "imperfect version of the game" (23). Where the algorithmic logic of the game is coherent and comprehensible, the real world seems messy and illogical. The text then, read as a creative work (on a 'meta' level), entreats its readers to critically approach the inability of the game to accurately reflect the real. The gamer as theorist is entreated to "be ludic, but lucid", to draw from the knowledge of the game, a knowledge of the game's structure - its algorithmic logic. It is only once the gamer becomes aware of this codification (takes the red pill) that he will be truly able to see the real's manifestation as game; and to critically engage therein (13).

\section{You are valuable}

Wark's text, while entreating us to take the red pill alludes to the temptation to take the blue pill, to disregard the structure and to allow oneself to to be consumed whole, captured in the pure agon. Games are fun; games are fair. Games reward equally and give us clear reasons for our failure. As such, games are seductive. We want to believe in the truth of an orderly system of advancement and reward. And yet, when we allow ourselves to be captured by the seductive illusion of equality presented by the game, we become the oysters of Wonderland, naively following the walrus towards our own eventual mass consumption.

This consumption, this capture is not only of ourselves, within the game, but it is of that which describes us as selves, our data. We are valuable both in our presence (as eyes, as clicks) and in our data (our 'likes', our age, our gender). Thus, it is the desire of the game to capture all of us so it might capture all that describes us. Carmen Hermosillo 
wrote about online bulletin boards (the original social networking interface) in 1994:

\section{I began to see that I had commodified myself. [...] I created my interior thoughts as a means of production for the corporation that owned a board I was posting to, and that commodity was being sold to other commodity consumer entities as entertainment. That means, I sold my soul like a tennis shoe and I derived no profit from the sale.}

As Hermosillo had begun to recognize, this narcissism-as-entertainment creates a profoundly weird relationship between the self, the second self, the other, and the corporation. The data that we wittingly and unwittingly sell to the board, the game, becomes a component of the game; it drives an iterative process of reflexive feedback wherein the rules of the game are modified to mirror our interests as they parallel the interests of the corporation or marketing firm. Our self-reported interests are aligned with those interests of the game and then fed back to us as a reflection of our innermost desires.

Here it becomes possible to identify a second type of cognitive dissonance that occurs due to the slippage between gamespace and gamic space, a dissonance which is situated in terms of our relationship to our avatar (or second self) as an invention of this process of consumption driven codification. The great promise of algorithmic logic has been increased efficiency, to allow us to become better versions of ourselves. Incentive-based systems (such as the fitness trackers) provide a means to objectively view our ordinary activity and a playful way by which we might optimize it. However, these applications also prompt the user to begin to see themselves not as their selves, but as the always-optimizable avatar version of the self created by the game and in the image of the game. Journalist Nora Young notes the oddity of this phenomenon: "Going about your daily life might become an exercise in performing to expectations. It changes the nature of human agency if I am not just behaving, but responding to an ideal image that I now must measure up to" (30). Young also notes: "It certainly feels as though we are inheritors of a very North American sense of self-improvement, conceived of as a kind of hygiene" (38). Through the perspective of this algorithmic extension of the protestant work ethic - the godly or hygienic drive towards optimization - every moment not devoted to productivity becomes wasted, dirty, and immoral. In our effort to achieve the idealized form of our avatars, we become beholden to the optimization $s /$ he is programmed to require. We become like a Sim, disengaged and robotically driven from one productive task to the next. Perhaps most disturbingly, this logic has begun to subsume not only the traditional space of work, but also that of leisure. Linkedln, Instagram, and Facebook are tools for the optimization of an algorithmically driven social engagement as prescribed by shadowy corporate interests.

\section{Do you wanna date my avatar?}

This strange relationship between the self and the avatar is explored in the comedic web series, The Guild. Created by and starring Felicia Day,[5] The Guild is plotted upon the initial awkwardness of a 'mmorpg'[6] guild (team of players) that must meet IRL (in real life). By jumping between traditional (3rd person) camera perspectives and confessionalstyle web-cam interactions, the series gives the viewer direct access to the juxtaposition of the idealized avatar-self and the messy 
real-self. "Do you Wanna Date My Avatar?" is one of a series of music videos incorporated into the series which ironically comment on, as well as punctuate, the narrative. It softly pokes fun at the disconnect between the real-life player and their game-world avatar, capitalizing on the temptation to withdraw from the imperfect 'real world' to the safe and perfect 'game world'. This juxtaposition is particularly potent considering the recent rise in popularity of online dating systems such as OkCupid and Tinder, which literally place users in the position of asking: "do you wanna date my avatar?". The playfulness with which the series - and this video specifically address the disconnect between the player and his/her avatar, who is "hotter than reality by far", invites the viewer to consider the seductive nature of the self as avatar and the strange disconnect that occurs when we aspire to achieve the perfection presented by our online, curated self.

Miranda July's application, Somebody, also explores the importance of authenticity and personal connection within an interconnected digital space. The application allows users to select physical proxies (other users) to act as (by proxy) message couriers; messages may include physical interactions (such as a hug) as well as text-based information. The associated marketing video for the application explores the possible absurdities of such 'by proxy' interactions through an increasingly surreal series of juxtapositions culminating in a sexual encounter that is interrupted by a plant requiring water. The work, by re-introducing the physical into a largely virtual interaction space, makes us examine the phenomenological one-ness of our primary and secondary selves. In doing so, it allows participants to critically examine the logic which undergirds our contemporary social interactions within virtual systems and to assess the validity of the algorithmic representation applied.

\section{Are you in or are you out?}

The drive towards algorithmic optimization within gamic space is intended as a virtual manifestation of our real-life projects of self improvement. The feedback that occurs as a result of this mirroring between the in-game and out-of-game selves manifests the final dissonance resulting from our ubiquitous adoption of game logic, the misaligned end-game.

Both Huizinga and Caillois agree that the purpose of play exists solely within the 'magic circle' of play. For Caillois, play creates "neither goods, nor wealth, nor new elements of any kind; and, except for the exchange of property among the players, [ends] in a situation identical to that prevailing at the beginning of the game" (10). Thus, the instrumentalization of play through the joint processes of gamification and the application of algorithmic structure is revealed to contain within it an inherent flaw which manifests in the conception of an end, or exit from the game. In gamespace, the way out of the game is built into the logic of the game; it is the point in which a winner or loser is declared and the game's self-contained purpose has been achieved. However, the process by which a player approaches this end is two-fold and contains within it an innate tension.

Walther describes this tension through distinguishing two modes of gamic interaction, playmode and gamemode. For Walther, playmode is that mode of interaction wherein players do not seek to progress through the game's structure (gain levels, follow the narrative) but instead seek only to remain playing, to remain in the space which is not ordinary. Gamemode, on the other hand, indicates the type of interaction wherein gamers are aware of the structure, the rules and actions they must take to progress towards the game's 
structurally manifested end-game. The tension between these two modes is a result of the disconnect between the desire to remain in gamespace indefinitely and a desire to win - which must motivate the continuation of play, but results ultimately in an exit from the game (Walther 256). This tension, reflected in our engagement with the ordinary made over as game, leads to anxiety as the invasion of the ordinary into the gamic space not only breaks the reverie of play but also brings to bear the actual, real-life end goal around which the game was (theoretically) focused. This is the point of failure for the simulation, the point from which we must choose to continue or not continue the process begun by the game without the comforting structure of the game.

This freedom creates an anxiety that arises from the misalignment of the endgames in gamic space and in the real, real world. There occurs a rupture between our understanding of the system's intention and the actual logic of the system which may not achieve the intended objective. This rupture breaks us out of the game and reveals the inadequacy of the technology in which we have placed our faith (the game).

This schism between the intentions of the game and the intentions of the player is revealed within Pham and Jamieson's Tinder hack. The hack's use of social engineering to acquire an absurd number of 'likes', which are highly unlikely to indicate real-world 'likes', renders an application that was initially designed as a tool for meeting people practically unusable towards that end. Despite our desire to believe that the Tinder-based end-game, becoming 'wanted' as indicated by the acquisition of likes, maps to becoming 'wanted' in real life, Pham and Jamieson's hack reveals that Tinder's simplified gamelogic is simply a mis-aligned end-game: The objective within Tinder does not result in the real-life optimization it promises.

\section{Players vs. Gamers, criti- cally addressing the gamic}

There is an established tradition in media arts of using the language of populist media (games, films) to critique the societies from which those media arise. This trend is exemplified by the website Molleindustria, an italian culture-jamming site that creates online flash games and machinima which critique current political and social trends. However, these critiques function not only on a narrative level, but also on a structural level. Molleindustria's management game, To Build A Better Mousetrap asks players (cats) to discover an 'ideal' management algorithm through the allocation of labour resources (mice) across a factory production model. In their machinima Welcome to the Desert of the Real (created from the Army recruitment game America's Army), images of murder (sniping) in a vast and desolate desert environment are juxtaposed with text from soldiers suffering from PTSD. In both of these situations, the radical juxtapositions of both narrative and structure ask the user/ player to critically address their simplified views of codified systems such as economics and war. Furthermore, they reinforce the distance between the avatar and the self. Particularly in the case of Welcome to the Desert of the Real, the real-world experience of soldiers is juxtaposed against the white-washed, detached experience of the war-game.

In Walther's gamemode, players move through the narrative of the game by reaching various in-game checkpoints and benchmarks required to progress. In doing this, they are not simply watching the game's narrative play out, they are exploring and learning the algorithmic rule space they must navigate and ultimately manipulate in 
order to win or to finish the game. Alexander Galloway explains this process of playing with a game while playing through it: "To play the game means to play the code of the game. To win means to know the system" (90-91). There is a distinction created between the average player and the serious gamer. Average players are content to progress through the narrative structure of the game. While they must learn the game logic (the means of play) they do not engage with it critically; they do not 'counter-game'. However, some players - as exemplified by Wark in Gamer Theory - prefer to interrogate the algorithm itself. These players co-opt the interface in an effort to discover meta-truths that are manifested within the imposed structure. While, in the case of To Build a Better Mousetrap, Molleindustria create a new game - one whose logic and structure reflect the incongruencies apparent in our acceptance of codified systems - their use of the already existent America's Army in Welcome to the Desert of the Real allows Molleindustria to develop their critique from within. This is the critical space held by the creative practices we have discussed. While the codified systems we use are frequently designed as tools, players must capitalize on the slippery signifiers that result from the application of game-like tropes to non-game activities (including gamification and codification) and fuzz up the boundaries between gamespace and gamic space. In doing so, players are able to reveal the cognitive fallacies that emerge from our acceptance of the codified logic.

Pham and Jamieson's hack exemplifies this critical approach by relying on user's trust of the Tinder system. For instance, Pham and Jamieson modified their profile pictures to appear 'sponsored' by Tinder. Users, as a result of this modification, assumed that the potential match was provided by the Tinder algorithm and dutifully 'swiped right', revealing that most of us are just average players. In this way, Pham and Jamieson utilize our trust in Tinder's algorithm to reveal the fallacy as well as the danger of such trust.

While it is critically necessary to question the validity of the codified systems we use, we also cannot escape them. When we extend these systems beyond those activities which are clearly games to those activities which have been made like a game (gamic), we realize that engaging as 'normal social individuals' requires our (at least partially) complicit participation. Thus, creative practitioners must interrogate these spaces from within. Like Huizinga's 'cheat', creative activists acknowledge 'the magic circle' while they subvert its internal logic, continuously critiquing its validity. These 'uber-users', who thrive within the slippage between gamespace and gamic space are forming a new class of creative critics that push us to continuously re-examine our datafied environment and our relationship to it while remaining fully implicated participants. 


\section{Notes}

[1] "Every job that must be done there is an element of fun. Find the fun, and snap! the job's a game!" (Mary Poppins).

[2] Despite the military roots of the headsup-display, its efficiency in presenting out-of-context meta-data in an immediately retrievable way and the ease with which it could be implemented in virtual game worlds have made it (for now) a visual trope associated with game worlds.

[3] This phenomenon could be viewed as largely unsurprising considering that Huizinga notes an innate function of play is that of civilization; culture, essentially is a manifestation of play, "is played from the very beginning" (52).

[4] Pervasive games are defined by Walther as games which utilize current technologies (such as GPS and WiFi) to create an augmented reality space in which the game is situated. The combination of computational structures in a post-screen setting is inherent to Walther's definition of this type of game (261). However, it is arguable that the current requirement to extensively prepare real-world spaces for the realization of this type of game is more a function of the as-yet-unrealized potential for ubiquitous adoption and coterminous integration of this technology into (at least) urban space and our own bodily space respectively and less a function of an inherent logic of the pervasive game structure. In other words, pervasive games might only require extensive technical preparation because we have yet to fully adopt their requisite technology. An example of this type of game would be the UK-based artist collective Blast Theory's game l'd Hide You, which was introduced in 2012 at the FutureEverything Festival in Manchester.

[5] Felicia Day is an internet/nerd-culture celebrity. She is an actress, producer and writer known for her reviews and commentaries on games/gaming as well as fantasy and science fiction literary culture.

[6] MMORPG stands for 'massive multiplayer online role-playing game', such as World of Warcraft or Everquest.

\section{Works cited}

Caillois, Roger. Man, Play, and Games. Urbana and Chicago: University of Illinois Press, 2001. Print.

Carroll, Lewis. Alice's Adventures in Wonderland. New York: MacMillan. 1865. Print.

Galloway, Alexander R. Gaming: Essays on Algorithmic Culture. Minneapolis, MN: University of Minnesota Press, 2006. Print.

Gray, Chris Hables. "Cyborging the Posthuman: Participatory Evolution." The Posthuman Condition. Ed. Kasper LippertRasmussen, Mads Rosendahl Thomsen, and Jacob Wamberg. Denmark: Aarhus University Press, 2012. 25-37. Print.

The Guild. Dir. Felicia Day. 2007-2012. Web Series. <http://watchtheguild.com/>

Hayles, Katherine. How We Became Posthuman. Chicago and London: University of Chicago Press, 1999. Print.

Huizinga, Johan. Homo Ludens: a study of the play element in culture. Boston: The Beacon Press, 1950. Print. 
Jamieson, Blake. "Beating the Tinder game. $800+$ Matches. I'll probably get banned for this..." 9 March 2014. Medium. Web (October 2014).<Medium.com>

Kirkpatrick, Graeme. Critical Technology: A social theory of personal computing. 2004. Electronic Publication.

The Matrix. Dir. The Wachowski Brothers. Perf. Keanu Reeves, Laurence Fishburne. Warner Bros, 1999. Film.

Andrews, Julie ."Just a Spoonful of Sugar." Mary Poppins. Composers Robert B. Sherman and Richard M. Sherman. Walt Disney Records, 1964. Musical, Soundtrack

Miranda July. Somebody. 2014. Mobile Application. <http://somebodyapp.com/>

Molleindustria. Welcome to the Desert of the Real. 2009. Machinima, video, web <http:// www.molleindustria.org/>

Molleindustria. To Build a Better Mousetrap. 2009. Web Application <http://www.molleindustria.org/>

okCupid. 2014. Web Application. <http:// www.okcupid.com/>

Pham, CamMi. "Cruel Intentions: How I Hacked Tinder and Got 2015 Matches in Under 17 Hours: The formula to become Wanted on Tinder." 18 April 2014. Medium. Web. <Medium.com>

The Sims. Electronic Arts Inc. 2014. Video Game.

Slavin, Kevin. "How Algorithms Shape our World." Online video. TED (filmed) July 2011. Web <TED.com>
Taleb, Nassim Nicholas. The Black Swan: the impact of the highly improbable. New York: Random House, 2007. Print.

Tinder. 2014. Mobile Application. <http:// www.gotinder.com/>

The Usual Suspects. Dir. Bryan Singer. Perf. Kevin Spacey, Gabriel Byrne, Chazz Palminteri, Stephen Baldwin, and Benecio del Toro. Polygram, 1995. Film.

Walther, Bo Kampmann. "Reflections of the Philosophy of Pervasive Gaming - with Special Emphasis on Rules, Gameplay, and Virtuality." Throughout: art and culture emerging with ubiquitous computing. Ed. Ulrik Ekman. Cambridge, MA: MIT Press, 2013.249-263. Print.

Wark, McKenzie. Gamer Theory. Harvard University Press, 2007. Electronic Publication.

Written? Kitten!. 2014. Web Application. <http://writtenkitten.net/>

Write or Die. 2014. Web Application. <http:// writeordie.com/>

Young, Nora. The Virtual Self: how our digital lives are altering the world around us. Canada: McClelland and Steward Ltd, 2012. Print. 